\title{
Social Anxiety in Orthognathic Patients
}

\author{
2 Fiona S Ryan (corresponding author) \\ 3 Consultant in Orthodontics \\ 4 Eastman Dental Hospital, UCLH, 256 Gray’s Inn Road, London, England, WC1X8LD. \\ $5 \quad$ Email: $\underline{\text { f.ryan@ucl.ac.uk }}$ \\ 6 Telephone: 02034561067 \\ 7
}

8 David R Moles

9 Professor of Oral Health Services Research/Director of Postgraduate Education and 10 Research/Specialist in Dental Public Health/Honorary Consultant in Dental Public Health 11 Peninsula Dental School, The John Bull Building, Research Way, Plymouth, Devon, 12 England, PL6 8BU.

13 Email: david.moles@plymouth.ac.uk

14

15 Justin T Shute

16 Consultant in Liaison Psychiatry / Honorary Lecturer

17 Orthodontic Department, Eastman Dental Hospital, 256 Grays Inn Road, London, WC1X 18 8LD.

19 Email: justin.shute@nhs.net

20

21 Alex Clarke

22 Consultant Clinical Psychologist

23 Royal Free Hospital, Pond St, London NW3 2QG.

24 Honorary Professor, Dept Clinical, Educational and Health psychology, UCL

25 Email: alexmacleodclarke@gmail.com

26

27 Susan J Cunningham

28 Professor of Orthodontics/Honorary Consultant

29 UCL Eastman Dental Institute, 256 Gray's Inn Road, London, England WC1X8LD. 
32 Funding:

33 This work was supported by grants from the European Orthodontic Society and the British

34 Orthodontic Society Foundation and supported by the UCLH/UCL Comprehensive 35 Biomedical Research Centre (CBRC), which receives funding from the Department of 36 Health NIHR funding scheme for BRCs.

37

38 Key words:

39 Social anxiety, orthognathic, fear of negative evaluation, psychology, dentofacial 40 deformity. 


\section{ABSTRACT}

43 There is evidence that patients seeking orthognathic treatment may be motivated by social

44 anxiety disorder (SAD). The aim of this study was to investigate SAD in orthognathic

45 patients using the Brief Fear of Negative Evaluation Scale (BFNES) and compare these

46 findings with the general population. This was a cross-sectional, questionnaire study

47 conducted in two parts. Firstly, a national survey was conducted to yield data for the

48 BFNES from a large, random sample of the UK general population. Secondly, orthognathic

49 patients completed the BFNES. The BFNES scores are reported in two formats; the original

50 12-item scale (O-BFNES) and a shorter 8-item version (S-BFNES). With regards to the

51 national survey, 1196 individuals participated. The mean O-BFNES score was 29.72 (SD

52 9.39) and S-BFNES score was 15.59 (SD 7.67). With regards to the orthognathic sample,

5361 patients participated. The mean O-BFNES score was 39.56 (SD 10.35) and mean S-

54 BFNES score was 24.21 (SD 8.41). Orthognathic patients had significantly higher scores

55 than the general UK population $(P<0.001)$ and multiple linear regression revealed that age,

56 gender, and patient status were all independent predictors of BFNES scores. From the

57 results of this study, orthognathic patients experience significantly higher levels of social 58 anxiety than the general population. 


\section{INTRODUCTION}

61 It has been estimated that approximately one in 100 people in the UK have a significant

62 visible facial defect, and that over 400,000 people will acquire a facial disfigurement in the

63 period of a year ${ }^{1}$. Concerns about physical appearance are often associated with social

64 anxiety, with individuals who perceive themselves as being unattractive exhibiting greater

65 levels of social anxiety ${ }^{2}$. This may result in problems in social interaction, leading to

66 lowered self-esteem and a tendency to become introverted and reclusive ${ }^{3}$. In addition, in a

67 clinical setting, individuals who seek surgical intervention for their problem may be

68 motivated by social anxiety and this could have negative implications for satisfaction and

69 psychological outcomes, as physical treatment may not alleviate psychological issues.

70 Social anxiety disorder (SAD) has been defined as 'an enduring fear of social situations

71 where the individual may be subject to evaluation by others ${ }^{, 4}$. It is the most common type

72 of anxiety disorder, with a prevalence of up to 18 per cent in the general population ${ }^{5}$. Fear

73 of negative evaluation is said to be the trademark of social anxiety, as this fear often leads

74 to an illogical and exaggerated anxiety in social situations ${ }^{6,7}$. This may be a factor in 75 orthognathic patients' motivation for treatment ${ }^{8}$.

76 Orthognathic patients have been shown to suffer from higher levels of state anxiety but

77 there is a paucity of information regarding social anxiety ${ }^{9}$. Indeed, there is only one

78 published study to date assessing the level of social anxiety in patients receiving

79 orthognathic treatment for non-cleft or craniofacial conditions ${ }^{10}$. The authors of that study

80 found that there was a small improvement in social avoidance and distress following

81 orthognathic treatment, but no statistically significant change in fear of negative evaluation. 
82 A small number of studies have investigated social anxiety and fear of negative evaluation

83 in patients with clefts and other types of facial deformity and generally found that patients

84 exhibited higher levels of social anxiety than unaffected groups ${ }^{11,12}$. Thus, the available

85 evidence suggests that patients who are visibly different, with either acquired or congenital

86 dentofacial conditions, may well exhibit higher levels of social anxiety than the general

87 population and this may have implications for treatment outcomes.

88 The aim of this study was to ascertain the extent and severity of fear of negative evaluation

89 in orthognathic patients compared with the general population. However there are limited

90 general population data available and the majority of these study samples have been

91 relatively small, restricted to college students/undergraduates, and have not been nationally

92 recruited; thus have limited generalisability ${ }^{13,14}$. Therefore a range of general population

93 values for the Brief Fear of Negative Evaluation Scale stratified on the basis of key

94 demographic data in a large, randomly recruited, national community population was

95 required first. The null hypothesis for this study was that there was no difference in mean

96 social anxiety, as measured by the Brief Fear of Negative Evaluation Scale, in orthognathic

97 patients and the general UK population.

98

\section{SUBJECTS AND METHODS}

\section{Instrument}

101 The Brief Fear of Negative Evaluation Scale (BFNES) measures the core construct in social 102 anxiety and is thought to be the most commonly used measure of social anxiety in clinical

103 studies $^{4,13,14}$. It is a self-report questionnaire, consisting of 12 items related to worrying or 
104 fearful $\operatorname{cognition}^{15}$. Eight of the items are positively scored and 4 are negatively scored, in 105 order to reduce the risk of response bias ${ }^{16}$. However, the reverse worded items have caused 106 some problems with the reliability and validity of the scale and, therefore, recent research 107 has suggested using the original 12 item scale (O-BFNES) but only including the 8 108 straightforward (S-BFNES) items in calculating the final score ${ }^{4,16}$. Despite the reservations 109 mentioned, most researchers continue to use the scale in its original format. This may be 110 because there are limited general population data available for the revised scale and this 111 restricts its use ${ }^{16}$.

\section{Part 1: General population sample}

\section{Participants}

115 Ethical approval for this study was granted by the relevant research ethics committee (ref: 116 2035/001). In order to obtain an unbiased, large, representative, random sample of the 117 general population, a national survey was conducted. This was undertaken via the Office 118 for National Statistics (ONS), which runs an omnibus survey in the UK called the Opinions 119 Survey. The ONS uses the Royal Mail's Postcode Address File to draw the sample and 120 over 2000 addresses are selected for each survey (17). This file contains the addresses for

121 approximately 27 million private households in the UK and is updated every three months.

122 It is the most up-to-date and complete address database in the $\mathrm{UK}^{17}$. By using this method 123 of random sampling, there is an equal chance of any individual being selected and thus bias 124 is reduced. Rigorous methodology was used to achieve the best possible response rate and 125 sample size, including making up to 8 attempts at face-to-face participant contact at 
126 different times of the day, followed by telephone contact. Participants were asked to 127 complete the Brief Fear of Negative Evaluation Scale (BFNES) questions themselves

128 (Appendix 1). Demographic data including age, gender, and ethnicity were also collected 129 during the survey.

\section{Part 2: Clinical Cohort}

131 Ethical approval was granted by the relevant research ethics committee (09/H0719/10). All 132 participants were recruited from one major teaching hospital site and had been accepted for 133 orthognathic treatment but had not yet commenced pre-surgical orthodontics. Inclusion 134 criteria were any patient undergoing combined orthodontics/orthognathic surgery, aged 16 135 years and over, and able to give informed consent. Exclusion criteria were patients with 136 congenital craniofacial anomalies (e.g. due to syndromes or clefts of the lip and/or palate), 137 patients with acquired facial defects, and those who had previously received orthognathic 138 treatment. As for the general population sample, patients were given the BFNES to 139 complete and demographic data, including age and gender, were also collected.

\section{Statistical analysis}

141 Statistical analysis was undertaken using the Statistical Package for Social Sciences 142 (version 19.0; SPSS Inc., Chicago, IL, USA). Demographic data were analysed 143 descriptively and the results from the 12-item scale (O-BFNES) and the 8-item 144 straightforward worded scale (S-BFNES) were tested for normality. All analyses were 145 conducted at the 0.05 level of significance. Comparisons between groups were made using 146 Student $t$-tests and one-way Analysis of Variance (ANOVA) with Bonferroni post-hoc 
147 tests. Multiple linear regression was undertaken to investigate the influence of group, age,

148 and gender on the BFNES score and to assess if there was an age/gender interaction.

149 The Opinions Survey data were weighted to correct for selection bias and non-response 150 bias. This weighting system has been developed by the Office for National Statistics based 151 on Census data. In addition, weightings were applied to the raw data to correct for response 152 bias. The weighted data were used for descriptive analyses in order to estimate population 153 parameters, whereas the unweighted data were used in the analytical statistical tests in order 154 to compare groups.

155 An a priori sample size calculation was performed using nQuery Advisor ${ }^{\odot}$ (version 7.0; 156 Statistical Solutions Ltd., MA, USA) using data from a similar study ${ }^{16}$. The calculation 157 assumed unequal sized groups, with an anticipated minimum of 1000 participants in the 158 general population group. This estimate was based on the minimum average response rate 159 for the monthly ONS Opinions surveys ${ }^{17}$. The clinically significant difference in the 160 BFNES scores was set at $10 \%$ of the total score based on clinical experience as there was 161 no supporting literature to guide this decision. A sample size of 31 orthognathic patients 162 was needed to detect a difference in means of $10 \%$ on the O-BFNES scale (4.8 points) 163 using an unpaired $t$-test with a power of $80 \%$ at the $5 \%$ level of significance. A sample size 164 of 46 orthognathic patients was needed to detect a difference in means of $10 \%$ on the S165 BFNES scale (3.2 points) using an unpaired $t$-test with a power of $80 \%$ at the $5 \%$ level of 166 significance. Therefore, it was decided to recruit a minimum of 50 orthognathic patients to 167 detect a clinically relevant difference for both scales allowing for some questionnaires to be 168 incorrectly completed or not returned. 


\section{RESULTS}

170

171 In total, 1196 individuals completed the survey, yielding a response rate of $66 \%$. As would

172 be expected in a population study using weighted data, the gender distribution was

173 approximately equal, with $51.1 \%$ females and $48.9 \%$ males. The largest age group was 25

174 to 44 year olds $(33.7 \%)$, with those over 75 years making up the smallest group (9.1\%).

175 Data on 18 different ethnic groups were collected. Summary statistics were calculated for

176 the BFNES for both the 12 item (O-BFNES) and 8 item (S-BFNES) scales. The weighted

177 mean, standard deviation, and range for the O-BFNES and S-BFNES stratified by age,

178 gender, and ethnicity are presented in Table 1. The overall mean score was 29.72 (SD 9.39)

179 for the O-BFNES and 15.59 (SD 7.57) for the S-BFNES.

180 Fear of negative evaluation was significantly higher in females than males, with a

181 difference of approximately 2 points on both scales $(P<0.001)$. Fear of negative evaluation

182 decreased with increasing age both in males and females and a univariate linear regression

183 model showed that for every ten year increase in age, fear of negative evaluation decreased

184 by one point when the genders were combined. The $\mathrm{R}^{2}$ values were 0.048 for O-BFNES, 185 and 0.057 for S-BFNES (Table 2). With regards to ethnicity, Gypsies/Irish Travellers

186 exhibited the highest BFNES scores and Chinese the lowest. However, due to the small 187 numbers within many subgroups, statistical analysis was not possible on the basis of the 18 188 different subgroups of ethnicity. Therefore, the ethnicity classification was collapsed into 189

British $(\mathrm{n}=1040)$ and non-British $(\mathrm{n}=154)$. One person answered 'don't know' and one 
190 refused to answer this section. There was no statistical difference in BFNES scores between

191 the British and non-British groups.

192 Part 2: Clinical cohort

193 In total, 61 orthognathic patients were recruited, $57.4 \%$ were female and $42.6 \%$ male. The 194 response rate was $100 \%$ and there were no missing data. The majority were in the 16 to 24 195 age group (50.8\%), followed by the 25 to 44 group (42.7\%). There were no patients above 196 the age of 64 years. The mean O-BFNES score for the whole patient group was 39.56 (SD 197 10.35) and the mean S-BFNES score was 24.21 (SD 8.41) (Table 3). A one-way analysis of 198 variance (ANOVA) revealed no evidence of a significant difference $(P=0.206)$ in BFNES 199 scores between the different age groups. Females had higher BFNES scores than males but 200 this was not statistically significant $(P=0.250$ for the O-BFNES and $P=0644$ for the S201 BFNES).

\section{Comparison of the general population sample and orthognathic patient data}

203 When comparing orthognathic patient data with the general population, orthognathic 204 patients had significantly higher fear of negative evaluation than the general population, 205 with statistically significant differences of almost 10 points for the O-BFNES and almost 206 nine points for the S-BFNES (Tables 4 and 5).

207 Multiple linear regression indicated that age, gender, and group (orthognathic patient or 208 general population participant) were all significant independent predictors of O-BFNES. 209 Orthognathic patients had O-BFNES scores that were 7.33 (95\% CI 4.83 to 9.84) higher on 210 average than the general population, having controlled for age and gender. With regards to 
211 the S-BFNES, again multiple linear regression indicated that age, gender, and group were

212 all significant independent predictors of S-BFNES. Orthognathic patients had S-BFNES

213 scores that were $6.38(95 \%$ CI 4.36 to 8.40$)$ higher on average than the general population,

214 having controlled for age and gender (Table 6).

215 Therefore, the null hypothesis that there is no difference in BFNES scores in orthognathic 216 patients and the general UK population was rejected.

\section{DISCUSSION}

218 The presence of FNE and the implications for clinical populations have not been 219 thoroughly investigated in orthognathic patients to date. The aim of this study was 220 therefore to investigate fear of negative evaluation in an orthognathic patient population. As 221 there were no general population data for FNE in the UK, it was necessary to initially 222 conduct a study to obtain these data.

223 The Brief Fear of Negative Evaluation Scale (BFNES) was used in this study (13). Due of 224 the lack of consensus about using the long or short form of the scale, the results of this 225 study were presented in both formats (O-BFNES and S-BFNES). This gives the reader and 226 other researchers the option of using either set of norms and to allow comparison with 227 previous published work using both scales.

228 The total mean BFNES score for the general population sample was 29.72 (SD 9.39) for the 229 O-BFNES and 15.59 (SD 7.67) for the S-BFNES. The closest comparable community 230 sample is from a US study of 489 volunteers recruited from a community volunteer registry 231 and the local university psychology department ${ }^{16}$. In this study, the total mean O-BFNES 
232 score was 30.55 and the S-BFNES was 15.91, which are similar to the results of the current

233 study. Duke and co-workers ${ }^{15}$ observed a higher mean O-BFNES score of 32.3 (SD 7.34) in

234 a community sample of 355 people recruited at religious meetings and in large retail centres

235 in the US, but the differences may be due to the more restricted sampling methodology

236 used.

237 Females exhibited significantly higher BFNES scores on both scales (2.12 points on the O-

238 BFNES and 1.73 on the S-BFNES) which is contrary to the findings of Rodebaugh and

239 colleagues who found no statistical difference in BFNES scores between the genders.

240 However, it must be borne in mind that the data from the Rodebaugh study was not

241 randomly or nationally obtained and thus may not be generalisable to the whole

242 population $^{16}$. In addition, females were over-represented in that study, with $72 \%$ of the

243 sample being female. The findings of the current study were in agreement with Duke and

244 co-workers who also found that scores were, on average, two points higher for females ${ }^{15}$.

245 The finding of higher social anxiety in females is in keeping with other published literature

246 that has found higher lifetime prevalence in females ${ }^{5,18,19,20}$.

247 There was a trend that BFNES score decreased with increasing age in the current study,

248 which is supported by the findings of Rodebaugh and colleagues ${ }^{16}$. However, the

249 magnitude of this effect was small, with a one point BFNES decrease for every decade

250 increase in age when the genders were combined, and this is unlikely to be clinically

251 relevant.

252 With regards to ethnicity, it was not possible to statistically examine the effects of each

253 different subgroup due to the small number of participants recruited in some of the 18 
254 different subgroups. Instead, the data were categorised into British origin and non-British

255 origin and there were no statistically significant differences between the two groups. This is

256 similar to the findings of a national US study on social anxiety disorders that found similar

257 ethnic distributions 5 .

258 The mean O-BFNES score for the whole patient group was 39.56 (SD 10.35) and the mean

259 S-BFNES score was 24.21 (SD 8.41). Female orthognathic patients had higher BFNES

260 scores than males but this was not statistically significant. The majority of studies

261 examining FNE and dentofacial deformity do not supply data on the effect of age and

262 gender, however, in the study on patients with amelogenesis imperfecta it was found that

263 females had higher BFNES scores than males but this difference was not statistically

264 significant ${ }^{21}$. It may be that the current study and the one by Coffield and co-workers

265 lacked the power to detect a significant difference between the genders if one existed. Age

266 had no statistically significant effect on BFNES scores for the orthognathic patients and this

267 is similar to the findings of Coffield and colleagues ${ }^{21}$. However, the age range in the current

268 study was narrow, and the sample size modest.

269 When the orthognathic patients were compared with the general population data, the 270 patients had significantly higher BFNES scores than the population values, with patient

271 scores eight to nine points higher than the general population. The only other study

272 assessing FNE directly in orthognathic patients found that patients had lower FNE than

273 norms, however, this study used the original 30-item Fear of Negative Evaluation Scale

274 (FNES) and thus the results are not directly comparable with those of this study ${ }^{10}$.

275 Additionally, their normative mean values were based on the US college sample from 
276 which the FNES was devised ${ }^{22}$. Another normative UK mean has been reported to be 14.26

277 (SD 7.72), however, this was also based on a student population and is probably not 278 representative of the general population ${ }^{23}$.

279 When comparing the two samples directly, multiple linear regression showed that age, 280 gender, and whether the participant was a patient or member of the general public were all 281 significant independent predictors of BFNES score. Orthognathic patients had a mean O282 BFNES score seven points higher and mean S-BFNES score 6 points higher than the 283 general population, having controlled for age and gender. Thus, we can conclude that the 284 orthognathic patients in this study did exhibit statistically significantly higher levels of fear 285 of negative evaluation than the general population. The magnitude of the difference is 286 considerable and it is likely to be clinically meaningful.

287 A clinically useful cut-off score of 38 when using the O-BFNES has been suggested by 288 Carleton and co-workers to diagnose social anxiety disorder ${ }^{4}$. When applied to the current 289 study results, $56 \%$ of the orthognathic patient cohort in this study met the criteria for a 290 positive diagnosis of social anxiety disorder (mean 39.56). However, the range of scores 291 reported was 16 to 60 and, thus, when examined on an individual basis, some patients did 292 not meet the cut-off point while others had very high scores. This variability has been 293 previously reported in populations with anxiety associated with disfigurement ${ }^{24}$ and 294 highlights the importance of examining each patient on an individual basis and not making 295 assumptions based on average values.

296 The limitations of this study should be borne in mind when interpreting the results. The 297 individuals in the general population sample were not screened for the presence of 
298 dentofacial deformity. This was due to the fact that the national survey was conducted by a 299 third party (Office for National Statistics) who have the resources and access to an 300 unbiased, representative sample of the UK general population. However, it was not possible 301 to train the surveyors to diagnose dentofacial discrepancy requiring orthognathic correction. 302 Therefore, it is possible that some potential orthognathic patients could have been recruited 303 into this sample. However, bearing in mind that the prevalence of dentofacial deformity is 304 relatively low and the sample was large, it is unlikely to have had a significant effect. In 305 addition, the orthognathic patients studied had higher BFNES scores than the general 306 population and thus their inclusion in the latter sample would only serve to underestimate 307 the actual difference in the populations studied. Another potential limitation of this research 308 was the relatively small size of the clinical sample, which precluded further in-depth 309 analysis. Future multicentre studies could be conducted to increase the generalisability of 310 the results.

311 Based on the results of this study and a review of the literature, it appears that the presence 312 of a facial disfigurement may be associated with elevated FNE and orthognathic patients 313 could be at increased risk of social anxiety disorder, regardless of age, gender, and severity 314 of the defect. Therefore, patients with facial disfigurement should be screened prior to 315 orthognathic treatment to assess baseline FNE levels, using the BFNES, which is quick and 316 acceptable to use chair-side. There is evidence that patients with visible facial 317 disfigurement with high FNE want psychological assistance and surgical correction alone 318 may not alleviate psychological symptoms ${ }^{25}$. A combination of cognitive behavioural 319 therapy and social skills training has been suggested to enable patients to develop a 
320 satisfactory body image and deal with others' evaluations ${ }^{25,26}$. Future longitudinal clinical

321 trials are needed to ascertain if FNE changes following orthognathic treatment and ideally

322 comparisons made with a similar group who are treated with psychological intervention

323 only. This study has established general population values for fear of negative evaluation,

324 as a measure of social anxiety, based on a large randomly selected general population

325 sample that can now be used in other studies.

326

\section{ACKNOWLEDGEMENTS}

328 We would like to thank the participants who gave up their time so freely and all members

329 of the orthognathic team. This work was supported by grants from the European

330 Orthodontic Society and the British Orthodontic Society Foundation and supported by the

$331 \mathrm{UCLH} / \mathrm{UCL}$ Comprehensive Biomedical Research Centre (CBRC), which receives funding

332 from the Department of Health NIHR funding scheme for BRCs. The funding bodies had 333 no role in the design of the study, data collection, analysis, or interpretation, report writing, 334 or decision to publish. The authors have no conflict of interest to declare.

\section{REFERENCES}

1. Partridge J, Julian D. The incidence and prevalence of disfigurement. Changing 338 Faces, London: 2008

339

2. Leary MR, Kowalski RM. Social Anxiety. Guilford Press, NY, 1995. 
3. Newell R, Marks I. Phobic nature of social difficulty in facially disfigured people. British Journal of Psychiatry 2000: 176: 177-181.

4. Carleton RN, Collimore KC, McCabe RE, Antony MM. (2011) Addressing revisions to the Brief Fear of Negative Evaluation scale: measuring fear of negative evaluation across anxiety and mood disorders. Journal of Anxiety Disorders 2011: 25(6): 822-828.

5. Kessler RC, Chiu WT, Demler O, Walters EE. (2005) Prevalence, severity, and comorbidity of 12-month DSM-IV disorders in the National Comorbidity Survey Replication. Archives of General Psychiatry 2005: 62: 617-627.

6. Rapee, R.M., Heimberg, R.G. (1997) A cognitive-behavioral model of anxiety in social phobia. Behaviour Research and Therapy, 35, 741-756.

7. Weeks, J.W., Heimberg, R.G., Fresco, D.M., Hart, T.A., Turk, C.L., Schneier, F.R., Liebowitz, M.R. (2005) Empirical validation and psychometric evaluation of the Brief Fear of Negative Evaluation Scale in patients with social anxiety disorder. Psychological Assessment, 17, 179-190.

8. Rumsey, N., Harcourt, D. (2004) Body image and disfigurement: issues and interventions. Body Image, 1, 1, 83-97.

9. Cunningham, S.J., Gilthorpe, M.S., Hunt, N.P. (2000) Are orthognathic patients different? European Journal of Orthodontics, 22, 195-202.

10. Lovius, B.B., Jones, R.B., Pospisil, O.A., Reid, D., Slade, P.D., Wynne, T.H. (1990) The specific psychosocial effects of orthognathic surgery. Journal of Craniomaxillofacial Surgery, 18, 8, 339-342. 
11. Berk, N.W., Cooper, M.E., Liu, Y.E., Marazita, M.L. (2001) Social anxiety in Chinese adults with oral-facial clefts. Cleft Palate and Craniofacial Journal, 38, 2, 126-133.

12. Versnel, S.L., Duivenvoorden, H.J., Passchier, J., Mathijssen, I.M. (2010) Satisfaction with facial appearance and its determinants in adults with severe congenital facial disfigurement: a case-referent study. Journal of Plastic and Reconstructive Aesthetic Surgery, 63, 10, 1642-1649.

13. Leary, M.R. (1983) A brief version of the Fear of Negative Evaluation Scale. Personality and Social Psychology Bulletin, 9, 371-375.

14. Rodebaugh, T.L., Woods, C.M., Thissen, D.M., Heimberg, R.G., Chambless, D.L., Rapee, R.M. (2004) More information from fewer questions: the factor structure and item properties of the original and Brief Fear of Negative Evaluation scale. Psychological Assessment, 16, 169-181.

15. Duke, D., Krishnan, M., Faith, M., Storch, E.A. (2006) The psychometric properties of the Brief Fear of Negative Evaluation Scale. Journal of Anxiety Disorders, 20, 6, $807-17$.

16. Rodebaugh, T.L., Heimberg, R.G., Brown, P.J., Fernandez, K.C., Blanco, C., Schneier, F.R., Liebowitz, M.R. (2011) More reasons to be straightforward: Findings and norms for two scales relevant to social anxiety. Journal of Anxiety Disorders, 25, 623-630.

17. Office for National Statistics. (2011) Opinions (Omnibus) Survey Information Guide. Office for National Statistics, London. 
18. Schneier, F.R., Johnson, J., Hornig, C.D., Liebowitz, M.R., Weissman, M.M. (1992) Social phobia: Comorbidity and morbidity in an epidemiological sample. Archives of General Psychiatry, 49, 282-291.

19. Offord, D.R., Boyle, M.H., Campbell, D.C., Goering, P., Lin, E., Wong, M., Racine, Y.A. (1996) One-year prevalence of psychiatric disorders in Ontarians 15 to 64 years of age. Canadian Journal of Psychiatry, 41, 559-563.

20. Ruscio, A.M., Brown, T.A., Chiu, W.T., Sareen, J., Stein, M.B., Kessler, R.C. (2008) Social fears and social phobia in the USA: results from the National Comorbidity Survey Replication. Psychological Medicine, 35, 15-28.

21. Coffield, K.D., Phillips, C., Brady, M., Roberts, M.W., Strauss, R.P., Wright, J.T. (2005) The psychosocial impact of developmental dental defects in people with hereditary amelogenesis imperfecta. Journal of the American Dental Association, $136,5,620-630$.

22. Watson, D., Friend, R. (1969) Measurement of social-evaluative anxiety. Journal of Consulting and Clinical Psychology, 33, 448-457.

23. Stopa, L., Clark, D.M. (2001) Social phobia: Comments on the viability of an analogue research strategy and British norms for the fear of negative evaluation questionnaire. Behavioural and Cognitive Psychotherapy, 29, 423-430.

24. Rumsey, N., Harcourt, D. (2012) The Oxford Handbook of the Psychology of Appearance. Oxford University Press, Oxford. 
404

405

406

407

408

409

410

411

25. Kent, G., Keohane, S. (2001) Social anxiety and disfigurement: The moderating effects of fear of negative evaluation and past experience. British Journal of Clinical Psychology, 40, 23-34.

26. Clarke, A., Thompson, A.R., Jenkinson, E., Rumsey, N., Newell, R. (2014) CBT for appearance anxiety: Psychosocial interventions for anxiety due to visible difference. Wiley Blackwell, UK. 


\section{TABLES}

413 Table 1. Population weighted means, standard deviations and ranges for the original 12 414 item BFNES (O-BFNES) and the straightforward 8 item (S-BFNES) from the ONS 415 Opinions survey within major classification categories.

\begin{tabular}{|c|c|c|c|c|c|c|}
\hline \multirow[t]{2}{*}{ Classification } & \multicolumn{3}{|c|}{ O-BFNES } & \multicolumn{3}{|c|}{ S-BFNES } \\
\hline & $\begin{array}{l}\text { Mean } \\
\text { score }\end{array}$ & SD & Range & $\begin{array}{l}\text { Mean } \\
\text { score }\end{array}$ & SD & Range \\
\hline All responders & 29.72 & 9.39 & $12-60$ & 15.59 & 7.67 & $8-40$ \\
\hline \multicolumn{7}{|l|}{ GENDER } \\
\hline Male & 28.64 & 8.84 & $12-59$ & 14.72 & 7.20 & $8-40$ \\
\hline Female & 30.76 & 9.79 & $12-60$ & 16.43 & 8.00 & $8-40$ \\
\hline \multicolumn{7}{|l|}{ AGE GROUP (in years) } \\
\hline 16 to 24 & 32.28 & 9.07 & $12-60$ & 17.62 & 7.99 & $8-40$ \\
\hline 25 to 44 & 31.03 & 10.22 & $12-60$ & 16.86 & 8.14 & $8-40$ \\
\hline 45 to 54 & 29.61 & 8.52 & $12-58$ & 15.48 & 6.95 & $8-40$ \\
\hline 55 to 64 & 28.65 & 9.06 & $12-57$ & 14.92 & 7.47 & $8-39$ \\
\hline 65 to 74 & 27.33 & 8.14 & $12-60$ & 12.80 & 6.49 & $8-40$ \\
\hline 75 and over & 25.44 & 7.71 & $12-55$ & 12.14 & 5.72 & $8-36$ \\
\hline \multicolumn{7}{|l|}{ ETHNICITY } \\
\hline $\begin{array}{l}\text { Combined (English, Welsh, Scottish, } \\
\text { Northern Irish, British) }\end{array}$ & 29.64 & 9.49 & $12-60$ & 15.55 & 7.73 & $8-40$ \\
\hline Irish & 26.58 & 8.05 & $14-45$ & 13.86 & 6.13 & $8-32$ \\
\hline Gypsy or Irish Traveller & 46.05 & 8.48 & $37-54$ & 27.92 & 6.49 & $21-34$ \\
\hline Any other White background & 30.91 & 10.13 & $12-52$ & 17.00 & 7.11 & $8-32$ \\
\hline White and Black Caribbean & 24.69 & 3.78 & $19-31$ & 10.59 & 2.88 & $8-18$ \\
\hline White and Black African & 30.11 & 0.88 & $29-31$ & 15.66 & 3.30 & $10-19$ \\
\hline
\end{tabular}




\begin{tabular}{|c|c|c|c|c|c|c|}
\hline White and Asian** & 29.62 & 7.88 & $12-34$ & 14.20 & 3.20 & $8-18$ \\
\hline $\begin{array}{l}\text { Any other mixed/multiple ethnic } \\
\text { background }\end{array}$ & 32.85 & 3.55 & $27-35$ & 17.46 & 0.89 & $16-18$ \\
\hline Indian & 29.10 & 9.03 & $12-44$ & 15.53 & 8.34 & $8-30$ \\
\hline Pakistani & 34.75 & 7.12 & $17-49$ & 18.70 & 6.86 & $8-32$ \\
\hline Bangladeshi & 30.32 & 7.01 & $17-34$ & 20.18 & 5.36 & $10-23$ \\
\hline Chinese & 23.53 & 1.31 & $22-25$ & 12.48 & 4.69 & 8-19 \\
\hline Any other Asian background & 30.03 & 5.90 & $20-43$ & 14.47 & 5.17 & $8-23$ \\
\hline African & 28.63 & 5.38 & $18-39$ & 15.40 & 3.82 & $9-22$ \\
\hline Caribbean & 29.76 & 5.67 & $19-42$ & 13.96 & 6.67 & $8-32$ \\
\hline $\begin{array}{l}\text { Any other Black/African/Caribbean } \\
\text { background }\end{array}$ & 30.81 & 8.65 & $16-42$ & 15.57 & 6.91 & $8-25$ \\
\hline Arab & 33.51 & 2.50 & $31-36$ & 14.52 & 3.50 & $11-18$ \\
\hline Any other ethnic group & 33.05 & 13.4 & $16-51$ & 18.63 & 10.45 & $8-34$ \\
\hline Refusal & 34.00 & $*$ & $34-34$ & 17.00 & $*$ & $17-17$ \\
\hline Do not know & 25.00 & $*$ & $25-25$ & 8.00 & $*$ & $8-8$ \\
\hline
\end{tabular}

*Standard deviation could not be calculated due to small sample number.

$418 * *$ Asian refers to individuals of South Asian descent. 
420 Table 2. Linear regression demonstrating effect of age on O-BFNES and S-BFNES scores.

421 [Note: Dependent variable: O-BFNES and S-BFNES respectively. Predictor: respondent's 422 age.

\begin{tabular}{|c|c|c|c|c|c|}
\hline Scale & Model & $\mathbf{R}^{2}$ & $\begin{array}{c}\begin{array}{c}\text { Age } \\
\text { coefficient }\end{array} \\
\text { (B) }\end{array}$ & $\begin{array}{l}\text { 95\% Confidence } \\
\text { interval of } B\end{array}$ & $P$-value \\
\hline O-BFNES & $\begin{array}{l}\text { (Constant) } \\
\text { Respondent's age }\end{array}$ & 0.048 & -0.114 & $\begin{array}{c}-0.144 \text { to } \\
-0.084\end{array}$ & $<0.001$ \\
\hline S-BFNES & $\begin{array}{l}\text { (Constant) } \\
\text { Respondent's age }\end{array}$ & 0.057 & -0.100 & $\begin{array}{c}-0.124 \text { to } \\
-0.076\end{array}$ & $<0.001$ \\
\hline
\end{tabular}

423

424 
425 Table 3. Means, standard deviations and ranges for the original BFNES (O-BFNES) and 426 the straightforward (S-BFNES) for the orthognathic patient cohort within major

427 classification categories.

\begin{tabular}{|l|c|c|c|c|c|c|c|}
\hline Classification & Number & \multicolumn{3}{|c|}{ O-BFNES } & \multicolumn{3}{|c|}{ S-BFNES } \\
\hline & & Mean & SD & Range & Mean & SD & Range \\
\hline All responders & 61 & 39.56 & 10.35 & $16-60$ & 24.21 & 8.41 & $8-40$ \\
\hline Gender & & & & & & & \\
\hline Male & 26 & 38.15 & 10.01 & $17-60$ & 24.04 & 7.88 & $8-40$ \\
\hline Female & 35 & 40.60 & 10.63 & $16-59$ & 24.34 & 8.90 & $8-39$ \\
\hline Age group & & & & & & & \\
\hline $\mathbf{1 6}$ to $\mathbf{2 4}$ & 31 & 41.16 & 10.40 & $16-57$ & 25.42 & 8.64 & $8-37$ \\
\hline $\mathbf{2 5}$ to 44 & 26 & 38.50 & 10.52 & $17-60$ & 23.35 & 8.26 & $8-40$ \\
\hline $\mathbf{4 5}$ to 54 & 3 & 35.33 & 9.07 & $27-45$ & 20.33 & 9.50 & $11-30$ \\
\hline $\mathbf{5 5}$ to $\mathbf{6 4}$ & 1 & 30.00 & $*$ & $30-30$ & 21.00 & 0.00 & $21-21$ \\
\hline
\end{tabular}

$428 \quad[*$ Standard deviation could not be calculated due to small sample number]. 
430 Table 4. Distribution of BFNES scores in the ONS Opinions Survey data and the 431 orthognathic study for the original 12-item BFNES (O-BFNES) and the straightforward 8432 item (S-BFNES).

\begin{tabular}{|l|c|c|c|c|c|c|}
\hline \multicolumn{3}{|c|}{ Classification } & \multicolumn{3}{|c}{ O-BFNES } & \multicolumn{3}{c|}{ S-BFNES } \\
\hline & Mean & SD & Range & Mean & SD & Range \\
\hline Orthognathic patients & 39.56 & 10.35 & $16-60$ & 24.21 & 8.41 & $8-40$ \\
\hline ONS survey & 29.72 & 9.39 & $12-60$ & 15.59 & 7.67 & $8-40$ \\
\hline
\end{tabular}

433

434 
435 Table 5. Comparison of BFNES scores between the ONS Opinions Survey data (ONS) and 436 the orthognathic patient data (OG) for the original 12-item BFNES (O-BFNES) and the 437 straightforward 8-item (S-BFNES).

\begin{tabular}{|l|c|c|c|c|c|c|}
\hline \multicolumn{1}{|c|}{ Scale } & Source & N & Score & $\begin{array}{c}\text { Mean } \\
\text { difference }\end{array}$ & $\begin{array}{c}\text { 95\% CI of } \\
\text { the mean } \\
\text { difference }\end{array}$ & P-value \\
\hline \multirow{2}{*}{ O-BFNES } & ONS & 1136 & 29.72 & -9.84 & $\begin{array}{c}-7.63 \text { to }- \\
12.58\end{array}$ & $<0.001$ \\
\hline & OG & 61 & 39.56 & & & \\
\hline \multirow{2}{*}{ S-BFNES } & ONS & 1149 & 15.59 & -8.62 & -6.78 to - & $<0.001$ \\
& OG & 61 & 24.21 & & 10.79 & \\
\hline
\end{tabular}

438

439 
440 Table 6. Multiple linear regression to assess the effect of group, age, and gender on O-

441 BFNES and S-BFNES.

\begin{tabular}{|c|c|c|c|c|c|}
\hline Scale & Model & $\mathbf{R}^{2}$ & $\begin{array}{c}\text { Coefficient } \\
\text { (B) }\end{array}$ & $95 \%$ CI of B & $P$-value \\
\hline O-BFNES & $\begin{array}{l}\text { (Constant) } \\
\text { Group } \\
\text { Respondent's age } \\
\text { Respondent's gender }\end{array}$ & 0.107 & $\begin{array}{r}-7.33 \\
-0.11 \\
2.10\end{array}$ & $\begin{array}{l}-9.84 \text { to }-4.83 \\
-0.14 \text { to }-0.09 \\
1.04 \text { to } 3.16\end{array}$ & $\begin{array}{l}<0.001 \\
<0.001 \\
<0.001\end{array}$ \\
\hline S-BFNES & $\begin{array}{l}\text { (Constant) } \\
\text { Group } \\
\text { Respondent's age } \\
\text { Respondent's gender }\end{array}$ & 0.119 & $\begin{array}{l}-6.38 \\
-0.10 \\
1.63\end{array}$ & $\begin{array}{l}-8.40 \text { to }-4.36 \\
-0.12 \text { to }-0.08 \\
0.78 \text { to } 2.49\end{array}$ & $\begin{array}{l}<0.001 \\
<0.001 \\
<0.001\end{array}$ \\
\hline
\end{tabular}

442 [Dependent variable: O-BFNES and S-BFNES respectively. Independent variables: Group

443 (survey participant or patient), respondent's gender, respondent's age]. 